\title{
An investigation into the structure and acquisition of orthographic knowledge: Evidence from cross-script Kanji-Hiragana priming
}

\author{
JEFFREY S. BOWERS \\ Rice University, Houston, Texas \\ and \\ YASUSHI MICHITA \\ University of the Ryukyus, Okinawa, Japan
}

\begin{abstract}
Numerous studies have demonstrated that orthographic knowledge is coded in an abstract format in English (e.g., the perceptually dissimilar words READ/read map onto a common abstract orthographic representation). However, it is unclear at present whether this mapping occurs at the letter or word level. Two experiments investigated this issue in a language (i.e., Japanese) in which words can be written in perceptually unrelated scripts (Kanji and Hiragana) and, crucially, in which there are no letter correspondences between scripts. Using the long-term priming paradigm, robust priming was obtained when study-test words were depicted in Hiragana-Kanji, and vice versa. Furthermore, little priming was obtained following a study-test modality shift. The modality-specific nature of this priming suggests that corresponding words in the two scripts share common orthographic representations. A model is outlined that describes how abstract orthographic knowledge is acquired.
\end{abstract}

A central issue for theories of reading concerns how letters and words are represented orthographically. That is, how are written materials perceptually coded within the visual system? According to most accounts, orthographic knowledge is coded in an abstract format in such a way that different exemplars of a given stimulus map onto the same representation (see, e.g., Coltheart, 1981; McClelland, 1976). For example, the visual patterns $A / a$ or $R E A D /$ read are thought to map onto common orthographic codes, despite the perceptual dissimilarity of the items. This is assumed to be the case even for items that are arbitrarily related in their perceptual form, as in the examples cited.

Evidence for the existence of abstract orthographic codes comes from a variety of sources. Coltheart (1981), for example, described a conduction aphasic patient who could not name pseudowords (e.g., nega), but who nevertheless was able to match uppercase/lowercase pseudowords that were perceptually dissimilar (e.g., NEGA/nega) without difficulty. Given that these items are (1) meaningless, (2) perceptually dissimilar in terms of uppercase/ lowercase, and (3) unpronounceable by the patient, Coltheart concluded that the patient accessed abstract orthographic codes in order to perform the task. Consistent with this conclusion, McClelland (1976) reported that the word superiority effect (WSE) is equally large for words pre-

Correspondence should be addressed to J. S. Bowers, Department of Psychology, Rice University, Houston, TX 77251-1892 (e-mail: bowers (@)rice.edu). sented in case-uniform and mixed-case conditions; for example, the words $F A D E$ and $f A d E$ were both better identified than the matched pseudowords $G A D E$ and gAdE in a task in which participants attempted to identify briefly presented targets. Given that mixed-case words are unfamiliar in their visual structure, these results suggest that the WSE is mediated by word representations coded in an abstract fashion (see also Bowers, Bub, \& Arguin, 1996). In addition, Bowers (1996) found longterm priming to be equally robust for words repeated in the same case and in different cases, even though the different-case words were perceptually dissimilar at study and test (e.g., READ/read). This cross-case priming was attributed to orthographic knowledge, since the priming was dramatically reduced following a study-test modality shift in which words were studied auditorily and tested visually (for additional evidence in support of the existence of abstract orthographic knowledge, see Besner, Coltheart, \& Davelaar, 1984, Bowers, Arguin, \& Bub, 1996, Evett \& Humphreys, 1981, and Rayner, McConkie, \& Zola, 1980, among others; but see Boles, 1992).

Taken together, these findings strongly support the conclusion that orthographic knowledge is coded abstractly. Under this assumption, two related questions need to be addressed: (1) Where within the orthographic system are these abstractions learned (i.e., the letter or word level)? (2) How are these codes acquired? Clearly, the answer to the first question would greatly constrain the possible solutions to the second; accordingly, it is first necessary to determine the specific loci at which abstractions take place. One possibility is that learning selectively occurs 
at the letter level, which would lead to abstract word codes, but only by virtue of letters serving as input to word representations. Alternatively, some learning procedure may operate at multiple levels, and accordingly, the existence of abstract word knowledge would not be contingent on, or necessarily the product of, abstract letter codes.

Current findings suggest that, at minimum, a learning process acts directly on letter representations to create abstract letter codes. Perhaps the best evidence in support of this conclusion is that the WSE extends to words presented in mixed-case letters (McClelland, 1976). Mixed-case words are unfamiliar (and unrecognizable) when considered as complete visual patterns, and the only familiar (and recognizable) features of the words are the component letters themselves. Thus, the WSE must reflect the activation of abstract letter codes, which in turn leads to the activation of abstract word codes (for additional evidence, see Besner \& Jolicoeur, 1997; Rynard \& Besner, 1987; for further discussion of this issue, see Bowers, Vigliocco, \& Haan, in press).

However, these and related findings cannot be used to determine whether learning is restricted to the letter level. The reason, of course, is that upper- and lowercase words are composed of upper-and lowercase letters, and, accordingly, any demonstration that words are coded abstractly can be attributed to learning that has taken place at the letter level. In order to avoid this ambiguity, it is necessary to determine the structure of word representations under a specific condition, namely when (1) words can be depicted in dissimilar formats and (2) the different formats do not share letter-by-letter correspondences. If these types of words map onto common orthographic codes, it must be concluded that the learning process interacts with word-level knowledge (since common abstract letter codes do not exist for these items).

In fact, a study by Brown, Sharma, and Kirsner (1984) provides some evidence compatible with this view. These authors assessed priming for Indian words displayed in Hindi-Urdu scripts: The spoken forms of these scripts are identical under normal circumstances, but their written forms are unrelated, and they do not share a simple letter-by-letter correspondence. Employing the lexical decision task, they observed similar (nonsignificantly dif- ferent) amounts of priming when items were studied and tested in the same (113-msec) and different $(93-\mathrm{msec})$ script conditions, consistent with the conclusion that Hindi-Urdu words map onto common orthographic word representations. It is important to note, however, that the authors did not compare the size of cross-script priming (which can be attributed to orthographic, semantic, or phonological codes) with cross-modal priming (which can be attributed to semantic or phonological codes), and, accordingly, the relative contribution of modalityspecific (orthographic) and modality-independent (nonorthographic) codes cannot be determined. In fact, Kirsner, Dunn, and Standen (1989) attributed this priming to phonological representations.

Two experiments are reported here that attempt to clarify this issue by comparing cross-script with cross-modal priming for words that do not share letter-by-letter correspondences in the two scripts; specifically, the Japanese scripts Kanji and Hiragana. Kanji characters (based on Chinese ideograms) represent morphemes, whereas Hiragana characters represent syllables, and the same words are depicted in both scripts. (Children tend to learn Hiragana first, and later Kanji.) In Experiment 1, test words were presented in Hiragana, and in Experiment 2, test words were presented in Kanji, providing an opportunity to assess cross-script priming in both the HiraganaKanji and Kanji-Hiragana study-test directions. See Figure 1 for a partial list of the experimental items used in Experiment 1. If robust cross-script priming is obtained in the face of minimal cross-modal priming, the priming would appear to be mediated by abstract orthographic word codes. ${ }^{1}$ The two experiments are described together.

\section{METHOD}

\section{Participants}

A group of 32 native Japanese speakers from the Houston and Madison areas volunteered to participate in Experiment 1 in exchange for $\$ 5$, and another group of 32 Japanese speakers from the University of Ryukyus volunteered to participate in Experiment 2.

\section{Design and Materials}

Experiments $1-2$ included a study-test condition as a within-subjects factor (study and test items presented in the same script vs. different scripts vs. different modalities vs. a nonstudied baseline condition). Different sets

$\begin{array}{lll}\text { Hiragana } & \text { Kanji } & \text { English Translation } \\ \text { やま } & \text { 山 } & \text { mountain } \\ \text { なつ } & \text { 夏 } & \text { summer } \\ \text { くつ } & \text { 蜮 } & \text { shoe } \\ \text { みず } & \text { 水 } & \text { water } \\ \text { おと } & \text { 音 } & \text { sound }\end{array}$

Figure 1. Examples of Hiragana and Kanji words used in Experiment 1, with English translations. 
of Kanji-Hiragana word pairs were used in the two experiments. In Experiment 1, the critical materials consisted of 64 common KanjiHiragana words and a set of 64 pronounceable Hiragana nonwords matched in length with the Hiragana words. In Experiment 2, the critical materials consisted of 64 common Kanji words composed of two Kanji characters (e.g., meaning music), the corresponding set of Hiragana words, and nonwords composed of two Kanji characters combined in a meaningless way (e.g., 酒). The latter words were selected from a set of the 2,000 most basic words in Japanese (National Language Research Institute, 1984). Homophones were excluded from both word lists.

In the study phase of both experiments, $16 \mathrm{Kanji}, 16$ Hiragana, and 16 spoken words were presented in random order, and at test, all 64 words and 64 nonwords were visually presented in random order. Four experimental files were created for each experiment so that each word was presented in each condition equally often, yielding counterbalanced designs.

\section{Procedure}

The procedure of the two experiments was similar. In the study phase participants read aloud all written words and repeated aloud all spoken words. Words were presented one at a time, every $4 \mathrm{sec}$. Immediately after completing the study phase, participants completed a lexical decision task. A practice set of 10 words and 10 nonwords was initially presented, followed by the critical items. In Experiment 1, participants pressed the right shift key of a computer keyboard as quickly as possible when the item was a word, and the left shift key otherwise. In Experiment 2, they pressed the right button of a mouse keypad if the item was a word, and the left button otherwise.

\section{RESULTS}

The lexical decision latencies and error rates in the various conditions of Experiments 1-2 are shown in Table 1. In Experiment 1, an analysis of the response latencies showed an effect of the study-test condition $\left[F(3,93)=7.79, M S_{\mathrm{e}}=969, p<.01\right]$, reflecting the robust priming in the repetition ( $31-\mathrm{msec})$ and cross-script (31-msec) conditions. Critically, a simple contrast revealed significantly more priming in the cross-script $(31-\mathrm{msec})$ than in the cross-modal $(10-\mathrm{msec})$ condition $[F(1,31)=$ $6.56, M S_{\mathrm{e}}=975, p<.05$ ], indicating that the cross-script priming was, in large part, modality specific. Indeed, the cross-modal priming was not significant $[F(1,31)=$ $\left.1.37, M S_{\mathrm{e}}=1,237, p>.25\right]$. The analysis of the error rates failed to reveal a main effect $[F(3,93)<1] .^{2}$

Table 1

\section{Mean Lexical Decision Latencies (RTs, in Milliseconds),}

Priming Scores (PS), and Error Rates as a Function of the Study-Test Condition in Experiments 1-2

\begin{tabular}{|c|c|c|c|c|}
\hline Study-Test Condition & $\mathrm{RT}$ & PS & $\%$ Errors & PS \\
\hline \multicolumn{5}{|c|}{ Experiment 1} \\
\hline Hiragana-Hiragana & 632 & 32 & 1.9 & 1.6 \\
\hline Kanji-Hiragana & 632 & 32 & 3.3 & 0.02 \\
\hline Spoken-Hiragana & 653 & 10 & 1.9 & 1.6 \\
\hline Nonstudied-Hiragana & 663 & & 3.5 & \\
\hline \multicolumn{5}{|c|}{ Experiment 2} \\
\hline Kanji-Kanji & 419 & 25 & 2.7 & 3.2 \\
\hline Hiragana-Kanji & 427 & 17 & 3.9 & 2.0 \\
\hline Spoken-Kanji & 441 & 03 & 6.1 & -0.2 \\
\hline Nonstudied-Kanji & 444 & & 5.9 & \\
\hline
\end{tabular}

Similar results were obtained in Experiment 2. An analysis of the response latencies showed a main effect of the study-test condition $\left[F(3,93)=11.13, M S_{\mathrm{e}}=386, p<\right.$ $.01]$, reflecting the robust priming in the repetition (25-msec) and cross-script (17-msec) conditions. A simple contrast revealed significantly more priming in the cross-script (17-msec) than in the cross-modal (3-msec) condition $\left[F(1,93)=7.78, M S_{\mathrm{e}}=386, p<.01\right]$, and again, the cross-modal priming was not significant $[F(1,93)<1]$. The analysis of the error rates revealed an effect of condition $\left[F(3,93)=3.13, M S_{\mathrm{e}}=.003, p<.05\right]$, reflecting the higher error rates in the baseline (.06) and cross-modal $(.06)$ conditions than in the repetition $(.03)$ and crossscript (.04) conditions.

\section{DISCUSSION}

The combination of robust cross-script and minimal (nonsignificant) cross-modal priming strongly suggests that corresponding Kanji and Hiragana words map onto common orthographic representations. These results extend the recent report of modality-specific cross-case priming in English (e.g., READ/read; Bowers, 1996) and suggest that abstract orthographic word codes can be acquired independentiy of abstract letter codes.

It is worth contrasting these cross-script and cross-case priming results with the null effects obtained between translation equivalents in bilingual speakers (see, e.g., Durgunoğlu \& Roediger, 1987; Kirsner, Smith, Lockhart, King, \& Jain, 1984) and the small or null priming effects obtained between heterographic homophones (e.g., sale/sail; Neisser, 1954; Ziemer \& Bowers, 1997). The latter results also demonstrate that semantic and phonological codes play a relatively small role in mediating long-term priming for visually presented test words; thus further support is provided for the conclusion that cross-script and cross-case priming are mediated by orthographic codes.

These findings raise the interesting question as to how abstract orthographic codes are acquired in the first place. That is, how does a perceptual system map together letters and words that are unrelated in their perceptual structure? In the following pages, we begin to outline one possible theory.

\section{How to Acquire Abstract Letter and Word Codes}

The Hiragana-Kanji priming results, in combination with findings supporting the existence of abstract letter codes (e.g., McClelland, 1976), suggest that the relevant learning processes directly interact with both letter and word codes during the development of abstract orthographic knowledge. Accordingly, learning is not restricted to a small set of letter codes, but instead extends to the much larger set of lexicalorthographic codes.

In considering the nature of this learning process, one conclusion seems necessary - namely, letter and word representations cannot be learned on the basis of the visual properties of the input. That is, bottom-up visual information cannot be used to map together arbitrarily related perceptual forms, such as the words $\$ / \mathrm{hi}$. Instead, it is necessary to assume that a nonvisual "teacher" acts on the orthographic system in a top-down fashion in order to organize the perceptual representations of both letters and words. ${ }^{3}$ This teacher would instruct the orthographic system to map together specific patterns (such as $A / a$ ) even though the items are perceptually unrelated.

One likely candidate for an external teacher is the phonological system, consistent with theories of reading that assume bidirectional connections between orthographic knowledge on the one hand, and phonological codes on the other (see, e.g., Grainger \& Ferrand, 1994; Van Orden, Pennington, \& Stone, 1990). To see how phonology could act as a teacher, consider two arbitrarily related visual letters (e.g., $A / a$ ), as depicted in Figure 2. In this figure, the two visual patterns map onto the same phonological representation, and because of the bidirectional con- 
nections between orthography and phonology, both orthographic patterns are consistently coactivated within the orthographic system, via feedback. It is this coactivation that makes it possible to learn arbitrary perceptual mappings. More specifically, the learning process might proceed as follows. When a child learns that visual pattern $A$ maps to sound /ei/, a bidirectional connection develops such that the presentation of $A$ leads to the activation of /ei/ and the presentation of /ei / activates $A$. Similarly, when the child learns that the visual pattern $a$ maps onto /ei/, bidirectional connections develop. As a result, when one of the visual patterns is presented-for example, $A$-it activates /ei/, which in turn activates $A$ and $a$, given the learned feedback connections. (Note that it is irrelevant whether the child learns the names for $A$ and $a$ at the same time. Coactivation of the visual patterns would be robust in either case.)

This coactivation, when combined with assoziative learning principles, provides a simple mechanism for acquiring abstract orthographic representations. For example, the Weber and associative decay rules described by Carpenter and Grossberg (1987; see also Bowers \& Vigliocco, 1997) would serve to associate representations that are consistently coactivated within the orthographic system, such as the letters $A / a$. These learning principles can readily account for the development of abstract orthographic codes between Kanji and Hiragana words, given that these dissimilar scripts contact the same lexical-phonological codes, which in turn would result in the consistent coactivation of both visual patterns each time one word is presented, via feedback.

It is important to note that there is independent evidence that orthographic and phonological codes are bidirectionally connected. The connections from orthography to phonology are not in dispute since these connections form the primary basis for reading aloud in most models of reading. In addition, there is also strong evidence for connections from phonology to orthography. For example, Dijkstra, Roelofs, and Fieuws (1995) reported that phoneme monitoring times for phonemes embedded in spoken words are affected by the spelling of the words, and Seidenberg and Tanenhaus (1979) reported that rhyme judgments of spoken words are more difficult when the words have dissimilar orthographies. For example, participants are faster to say that lie and tie rhyme than rye and tie. In both of these examples, the orthographic properties of the materials are irrelevant to the task demands, but these codes are nevertheless contacted and affect performance. More recently, Stone, Vanhoy, and Van Orden (1997) reported that visual lexical decisions are affected not only by the consistency with which a word's orthographic structure maps onto phonology, but also by the consistency with which its phonological structure maps onto orthography, with slower lexical decisions obtained for words with bidirectional inconsistencies. Taken together, these findings strongly support the conclusion that bidirectional connections exist between orthographic and phonological representations and lend support to the hypothesis that phonological codes act as teacher in constructing abstract orthographic representations.

In the example provided, phonological codes play the role of teacher, but it is also possible that bidirectional connections exist between orthographic and lexical-semantic representations and that these latter codes also contribute to the development of abstract orthographic representations. One finding compatible with this claim is that heterographic homophones (e.g., sail/sale) do not map onto common abstract orthographic codes (as demonstrated, among other findings, by the small or nonexistent long-term priming obtained between heterographic homophones; Neisser, 1954; Ziemer \& Bowers, 1997). One possibility is that although these items receive consistent feedback from common lexical-phonological codes, they do not receive consistent feedback from lexical-semantic codes, which prevents the development of abstract orthographic knowledge. Thus, on this account, abstractions between perceptually unrelated words take place only when the orthographic system receives consistent feedback from both phonological and lexical-semantic codes.

Additional evidence compatible with the view that lexical-semantic codes assist in the role of teacher comes from studies indicating that orthographic representations encode morphological relations, at least when the items are regularly related in their phonological form (e.g., guest/guests). Morphological relations are defined on semantic criterion, and it is often suggested that morphological relatives (at least inflections) share common lexical-semantic codes (see, e.g., Levelt, 1989). One possible explanation for the development of morphological structure within orthography is that different inflectionally related words receive feedback from common lexical-semantic codes, which in turn leads to the consistent coactivation of these orthographic patterns. Furthermore, if consistent feedback is required from both semantic and phonological codes, it might be predicted that the orthographic system would not map together irregularly related items, such as goose/geese. Indeed, it has been argued that orthographic representations encode only regular relations (see, e.g., Caramazza, Laudanna, \& Romani, 1988). On the present argument, then, the same learning principles that account for the development of abstract cross-case and cross-script representations also explain the development of morphological structure within the orthographic system.

To summarize, according to the present hypothesis, semantic and phonological representations act together as teacher to support the

\section{Orthographic Codes}

\section{Phonological Codes}

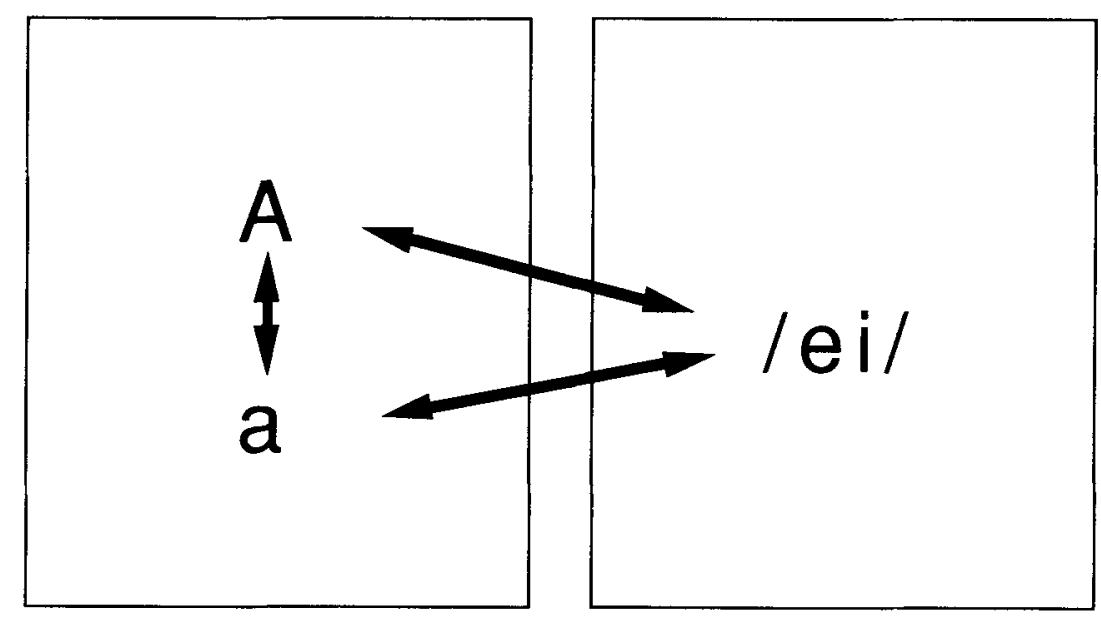

Figure 2. The visual patterns $A$ and $a$ each map onto the phonological code /ei/, which results in the coactivation of $A$ and $a$ each time one of the patterns is presented, via feedback. Associative learning principles within the orthographic system act to map together these coactive patterns to produce an abstract letter code. 
learning of abstract orthographic codes. Note that we are not claiming that phonological or semantic information is represented internally to the orthographic system. Rather, these codes provide the feedback that results in the consistent coactivation of orthographic patterns (such as $a / A$ ), and then associative learning within the orthographic system maps these items together to form abstract orthographic codes.

Before concluding this discussion, we should address two possible points of confusion. First, the empirical finding that long-term priming is largely modality specific may appear to be at odds with the claim that bidirectional connections exist between phonological and orthographic knowledge (and between semantic and orthographic knowledge). This theory might seem to predict robust cross-modal priming. However, theory and data can be reconciled if it is assumed that long-term priming is greatly reduced between associated as compared with repeated studytest items. Indeed, the empirical findings seem to require this assumption, not only to account for the reduction of cross-modal priming, but also to account for the small or nonexistent long-term priming obtained between synonyms and written associates (e.g., elephant-tusk; Roediger \& Challis, 1992). Thus on the present theory, complete priming is obtained between Kanji and Hiragana words because they gain repeated and direct access to the same orthographic representations. By contrast reduced priming is obtained between spoken and written words (and semantic associates) because the study-test items gain access only to the same representations indirectly though association.

Second, the claim that different visual instances of letters and words are coactive each time a specific item is studied might seem to render the development of abstract orthographic codes superfluous. For example, if studying the lowercase word garden leads to the coactivation of the orthographic patterns garden and GARDEN, these separate representations might individually support priming when garden or GARDEN is repeated at test. On this argument, then, priming would occur without positing abstract orthographic codes. However, this interpretation runs into trouble because it attempts to explain long-term priming on the basis of associative effects - and, as noted, long-term associative priming tends to be much reduced compared with repetition priming. In addition, this characterization of orthographic knowledge runs into empirical difficulties in other domains. For example, as noted in the introduction, some dyslexic patients cannot access phonology from print, but they can nevertheless match visually dissimilar upper- and lowercase letter strings that are meaningless (e.g., NEGA/nega; Coltheart 1981). Given that phonology and semantics cannot support the coactivation of these orthographic patterns via feedback, it is difficult to explain this result without positing abstract letter codes. ${ }^{4}$

\section{Implications for Modularity}

On a closing note, it is worth mentioning the implications of the present finding for theories of modularity. One of the basic premises of modular theories of perception is that the representations internal to a given system are insulated from other types of information. For example, the perceptual processes involved in identifying the written word table are presumed to be unaffected by our background knowledge of meaning and phonology. However, the present finding suggests that orthographic representations are structured in such a way that they are compatible with our background knowledge (e.g., that Kanji-Hiragana words share the same meaning and the same name, and that car/cars are inflectionally related), even though this structure cannot be determined on the basis of the perceptual attributes of the words themselves. On the present argument, this structure is learned through a penetration of lexical-semantic and phonological information into the orthographic system, contrary to a modular view of word perception. Whether or not this specific characterization of the learning process is correct, some type of top-down teacher seems required, and any such teacher violates the view that visual word recognition takes place within a modular system.

Perhaps an advocate of modular theories would object, saying that modularity is a theory of on-line perceptual processing, not a theory of perceptual learning. That is, it might be claimed that the learning processes themselves are nonmodular, but once the various perceptual systems are set up, the identification of words (or whatever else) takes place in a modular fashion. Still, one is left with the dilemma of how a nonmodular learning procedure can operate in the context of a modular theory of on-line perceptual processing. The present characterization of word processing certainly does not fit this account, given that the learning was dependent on semantic and phonological information consistently activating orthographic representations. Thus, a challenge for the modularist, it seems, is whether a motivated distinction can be drawn between nonmodular learning and modular on-line perceptual processing. This is clearly an interesting question that merits further study.

\section{REFERENCES}

Besner, D., Coltheart, M., \& Davelaar, E. (1984). Basic processes in reading: Computation of abstract letter identities. Canadian Journal of Psychology, 38, 126-134.

BESNER, D., \& JOLICOEUR, P. (1997). Basic processes in reading: Crosscase letter matching without phonology. Manuscript submitted for publication.

BoLEs, D. B. (1992). Fast visual generation: Its nature and chronometrics. Perception \& Psychophysics, 51, 239-246.

BowERS, J. S. (1996). Different perceptual codes support word and pseudoword priming: Was Morton right all along? Journal of Experimental Psychology: Learning, Memory, \& Cognition, 22, 1336-1353.

BowERS, J. S., ARGUIN, M., \& BUB, D. (1996). Fast and specific access to orthographic knowledge in a case of letter-by-letter surface alexia. Cognitive Neuropsychology, 13, 525-567.

BowERS, J. S., BUB, D., \& ARGUIN, M. (1996). A characterization of the word superiority effect in a case of letter-by-letter surface alexia. Cognitive Neuropsychology, 13, 415-441.

Bowers, J. S., \& VIGLIOcco, G. (1997). Grossberg and colleagues solved the hyperonym-hyponym problem over a decade ago. Manuscript submitted for publication.

Bowers, J. S., VIGliocco, G., \& HAaN, R. (in press). Orthographic, phonological, and articulatory contributions to masked letter and word priming. Journal of Experimental Psychology: Human Perception \& Performance.

Brown, H. L., Sharma, N. K., \& Kirsner, K. (1984). The role of script and phonology in lexical representation. Quarterly Journal of Experimental Psychology, 36A, 491-505.

Caramazza, A., Laudanna, A., \& Romani, C. (1988). Lexical access and inflectional morphology. Cognition, 28, 297-332.

Carpenter, G., \& Grossberg, S. (1987). A massively parallel architecture for a self-organizing neural pattern recognition machine. Computer, Vision, Graphics, \& Image Processing, 37, 54-115.

COLTHEART, M. (1981). Disorders of reading and their implications for models of normal reading. Visible Language, 3, 245-286.

Dukstra, T., Roelofs, A., \& Fieuws, S. (1995). Orthographic effects on phoneme monitoring. Canadian Journal of Experimental Psychology, 49, 264-271.

DURGUNoĞLU, A. Y., \& RoEdiger, H. L., III (1987), Test differences in accessing bilingual memory. Journal of Memory \& Language, 26, $377-391$

Evett, L. J., \& Humphreys, G. W. (1981). The use of abstract graphemic information in lexical access. Quarterly Journal of Experimental Psychology, 33A, 325-350.

Grainger, J., \& Ferrand, L. (1994). Phonology and orthography in visual word recognition: Effects of masked homophone primes. Journal of Memory \& Language, 33, 218-233.

Kirsner, K., Dunn, J. C., \& Standen, P. (1989). Domain-specific resources in word recognition. In S. Lewandowsky, J. C. Dunn, \& K. Kirsner (Eds.), Implicit memory: Theoretical issues (pp. 99-122). Hillsdale, NJ: Erlbaum.

Kirsner, K., Smith, M. C., Lockhart, R. S., King, M.-L., \& JaIN, M. (1984). The bilingual lexicon: Language-specific effects in an integrated network. Journal of Verbal Learning \& Verbal Behavior, 23; 519-539.

KomatsU, S.-I., \& Naito, M. (1992). Repetition priming with Japanese Kana scripts in word-fragment completion. Memory \& Cognition, 20, 160-170.

LEVELT, W. (1989). Speaking: From intention to articulation . Cambridge, MA: MIT Press.

MCClelland, J. L. (1976). Preliminary letter identification in the perception of words and nonwords. Journal of Experimental Psychology: Human Perception \& Performance, 3, 80-91. 
National Language Research InStitute (1984). A study of the fundamental vocabulary for Japanese language teaching. Tokyo: Shuei Shuppan.

NeISSER, U. (1954). An experimental distinction between perceptual processes and verbal response. Journal of Experimental Psychology, 47, 399-402.

PolK, T. A., \& FARAH, M. J. (1997). A simple common contexts explanation for the development of abstract letter identities. Neural Computation, 9, 1277-1289.

RAYNER, K., MCCONKIE, G. W., \& ZOLA, D. (1980). Integrating information across eye movements. Cognitive Psychology, 12, 206-226.

Roediger, H. L., III, \& Challis, B. H. (1992). Effects of exact repetition and conceptual repetition on free recall and rimed word-fragment completion. Journal of Experimental Psychology: Human Learning \& Memory, 18, 3-14.

RYNARD, D., \& BESNER, D. (1987). Basic processes in reading: On the development of cross-case letter matching without reference to phonology. Bulletin of the Psychonomic Society, 25, 361-363.

Seidenberg, M. S., \& Tanenhaus, M. K. (1979). Orthographic effects on rhyme monitoring. Journal of Experimental Psychology: Human Learning \& Memory, 5, 546-554.

Stone, G. O., Vanhoy, M., \& Van Orden, G. C. (1997). Perception is a two-way street: Feedforward and feedback phonology in visual word recognition. Journal of Memory \& Language, 36, 337-359.

Van Orden, G., Pennington, B., \& Stone, G. (1990). Word identification in reading and the promise of subsymbolic psycholinguistics. Psychological Review, 97, 488-522.

ZIEMER, H., \& BOWERS, J. S. (1997). Comparing phonological priming in three implicit memory tests. Poster presented at the Southwestern Psychological Association.

\section{NOTES}

1. Komatsu and Naito (1992) assessed priming between Hiragana and Katakana words in Japanese and reported that the cross-script priming was no larger than the cross-modal priming. It is important to note, however, that Katakana and Hiragana are used to represent foreign loan and common words, respectively, and thus Japanese readers do not have any experience reading the same word in the two scripts. Accordingly, it is not surprising that abstract orthographic codes do not develop between these items. By contrast, Kanji and Hiragana scripts depict the same set of words, and thus abstract orthographic codes should develop between these scripts if learning takes place at the word level.
2. Although the error rates in the various conditions did not differ significantly from one another, there was a slight trend for more errors in the cross-script (3.3) than in the cross-modal (1.9) condition, which might raise concerns that the greater RT priming in the cross-script (31-msec) compared with the cross-modal $(10-\mathrm{msec})$ condition was the product, at least in part, of a speed-accuracy tradeoff. In an attempt to evaluate this possibility, we removed from each of the four counterbalanced files the results of the participant who had the largest discrepancy in error rates in these two conditions, with more errors in the cross-script condition. By this criterion, we were able to unambiguously remove 1 participant from each file and to recompute the error and RT data. Not surprisingly, after removing the results of these 4 participants, the error rates in these two conditions were similar, with slightly fewer errors in the cross-script $(3.10 \%)$ than in the cross-modal (3.77\%) condition. Critically, the pattern of the RT data remained unchanged, with $27-\mathrm{msec}$ priming in both the repetition and cross-script conditions, and 6-msec priming in the cross-modal condition, indicating that the RT priming results cannot be attributed to a speed-accuracy tradeoff.

3. Polk and Farah (1997) have advanced an account of how abstract letter codes might be acquired in a bottom-up account in English. They have argued that most letters are visually similar in upper- and lowercase and these items provide a context in which abstract codes can be acquired for visually dissimilar letters. So, although $A / a$ cannot be mapped together in a bottom-up fashion when considered in isolation, these items are learned in the context of words that are similar overall, such as $C A P / c a p$. Thus Polk and Farah have suggested that abstract letter codes are acquired through the context of the similar letters. They in fact described a simple Hebbian learning model that does learn abstract letter identities through the use of similar letter contexts. However, this cannot be the complete story, since this approach cannot account for the development of orthographic codes that map together Kanji-Hiragana words. For these words, there are no similar contexts that could provide a basis for bootstrapping. This theory also ignores the various results suggesting that phonological codes activate orthographic representations during reading, via feedback (e.g., Stone, Vanhoy, \& Van Orden, 1997).

4. On the present theory, abstract orthographic codes cannot be acquired without consistent feedback from phonological and semantic representations. However, after these abstract codes are learned, the feedback is no longer necessary in order to match different versions of the same letter or word.

(Manuscript received May 8, 1997; revision accepted for publication October 1, 1997.) 